\title{
Immunomodulatory Effects of Eisenia bicyclis on Innate Immune Cells in Acute Exercise-Stress Rat Model
}

\author{
Seung Namkoong ${ }^{1}$, Eui-Su Cheoung ${ }^{2}$, Haemi Joo ${ }^{3}$, Seon-A Jang ${ }^{3}$, Yoon-Jung Yang ${ }^{4}$, \\ Se-Chan Kang ${ }^{4}$, Ki-Hyo Jang ${ }^{5}$ and Eun-Hwa Sohn ${ }^{6}$ * \\ ${ }^{1}$ Department of Physical Therapy, Kangwon National University, Samcheok 245-907, Korea, ${ }^{2}$ DanjeongBio Co., \\ Ltd., Wonju 220-710, Korea, ${ }^{3}$ Radiation Health Research Institute, Korea Hydro \& Nuclear Power Co., Ltd, \\ Seoul 132-703, Korea, ${ }^{4}$ Department of Natural Medicine Resources, Semyung University, Jecheon 309-711, \\ Korea, ${ }^{5}$ Department of Food and Nutrition, ${ }^{6}$ Department of Herbal Medicine Resource, Kangwon National \\ University, Samcheok 245-907, Korea
}

\begin{abstract}
In this study we investigated effects of supplementation with ethyl acetate extracts of the brown alga Eisenia bicyclis on innate immune cells to evaluate the possibilities as an immunomoulator in exercise stress. Twenty male SD rats were divided into four groups and the treatments were as follows: A, no Eisenia bicyclis extract (EBE) $(200 \mathrm{mg} / \mathrm{kg})$ intake and maintained at rest ; B, no EBE intake and undergoing exercise ; C, EBE intake and undergoing exercise ; D, EBE intake and maintained at rest. After 5 weeks of oral supplementation, rats were undergoing intensive swimming exercises for $2 \mathrm{~h}$ and sacrificed to assess the effects on peritoneal macrophages, spleen cells and natural killer (NK) cells. We showed increasing effects on nitric oxide-inducible nitric oxide synthase (NO-iNOS) production by macrophages and no effects of NK tumoricidal activity and suppressive effects on spleen cell proliferation in exercise group. However, EBE supplementation suppressed NO-iNOS production by macrophages and increased NK tumoricidal activity and spleen cell proliferative response to mitogen in exercise group. Overall, these results that EBE supplementation has differential effects on innate immune response and could be useful as sports nutrition.
\end{abstract}

Key words - Eisenia bicyclis, Exercise, Macrophage, NK cell, Proliferative response

\section{Introduction}

Marine environment has been the source of diverse life forms that produce different biologically active compounds. Metabolites from marine plants have been reported to have outstanding biological activities and profound applications in nutraceuticals, cosmeceuticals, and pharmaceuticals. Eisenia bicyclis is a species of the kelp, a member of the brown algae group of seaweeds, best known for its use in Asia. Eisenia bicyclis is high in calcium, iodine, iron, magnesium, and vitamin A as well as being a good dietary source for many other minerals. They also contain the storage polysaccharide laminarin, alginate, fucoxanthin and fucoidan with immunological activities (Joe et al., 2006). It has been also known that brown algae extracts closely linked to its protective properties against free radical attack and have anti-oxidant effects $\mathrm{Hu}$

${ }^{*}$ Corresponding author. E-mail : ehson@kangwon.ac.kr et al., 2010; Kang et al., 2004).

The innate immune system represents the first line of defense against microorganisms as it promptly initiates a local inflammatory reaction. Macrophages and NK cells are important components of the innate immune system and may play an important role in protection against acute and chronic virus infections, early recognition of tumor cells, and tumor spread (Adams and Hamilton, 1984; Whiteside and Herberman, 1989).

Many studies have demonstrated that exercise induces considerable physiological change in the immune system (Pedersen and Hoffman-Goetz, 2000). It has been suggested that exercise represents a quantifiable model of physical stress because many clinical physical stressors (e.g. surgery, trauma, burn and sepsis) induce a pattern of hormonal and immunological responses that have similarities to that of exercise (Hoffman-Goetz et al., 1994; Madden and Felten, 1995). Other studies show that several inflammatory cytokines 
can be detected in plasma during and after strenuous exercise (Ostrowski et al., 1998a; Ostrowski et al., 1999). The strenuous exercise induces an increase in the proinflammatory cytokines TNF- $\alpha$ and IL-1 $\beta$ and a dramatic increase in the inflammation responsive cytokine IL-6. The presence of multiple cytokines (TNF-a, IL-1 $\beta$, IL-6, IL-2 receptors, and IFN- $\gamma$ ) in urine after exercise shows that the expression of a broad spectrum of cytokines in response to exercise is possible (Sprenger et al., 1992).

To our knowledge, the immunomodulatory effects of EBE on innate immune system are still unknown and the effects of the EBE as sports nutrition for ameliorating acute exerciseinducing inflammation has not been clarified yet in animal studies. Therefore, in this study we focused on the immunomodulatory effects of EBE fraction on innate immune system underlying exercise-induced immune system.

\section{Materials and Methods}

\section{Preparation of ethyl acetate extract}

The fronds of Eisenia bicyclis were harvested from the coast of Ulleung Island, East Sea, South Korea in June 2009. Fresh Eisenia bicyclis was washed three times with tap water to remove salt and impurities, then dried at room temperature for 3 days and stored at $-20^{\circ} \mathrm{C}$. The dried samples were homogenized, using a grinder, before extraction. Dried Eisenia bicyclis powder (10 g) was dissolved in 70\% methanol (200 $\mathrm{ml}$ ) and shaken for $24 \mathrm{~h}$. After the extraction, the supernatant was recovered by centrifugation $(7000 \mathrm{rpm})$ at $4{ }^{\circ} \mathrm{C}$ for $30 \mathrm{~min}$ and the methanol was evaporated using a rotary vacuum evaporator (Tokyo Rikakikai Co. Ltd., Tokyo, Japan). The extract powder was suspended in distilled water and then partitioned with n-hexane, methylene chloride, and ethyl acetate $(\mathrm{EtOAc})$ in sequence. EtOAc fraction was evaporated and kept on $-20^{\circ} \mathrm{C}$ until used.

\section{Experimental animals}

Male SD rats $(n=24)$ were purchased from Joongang Experimental Animal Co. (Seoul, Korea) at 5 weeks of age, and the six animals were housed together in one cage in controlled environment under a light-dark cycle (lights on at 19:00 and off at 7:00). The experimental procedures were conducted according to the Code of Ethics for Animal Experimentation of Semyung University. After 1 week acclimation, all rats were randomly divided into four groups and the treatments were as follows: A, no EBE $(200 \mathrm{mg} / \mathrm{kg})$ intake and maintained at rest ; $\mathrm{B}$, no EBE intake and undergoing exercise ; C, EBE intake and undergoing exercise ; D, EBE intake and maintained at rest. After 5 weeks for oral supplementation, rats were undergoing intensive swimming exercises for $2 \mathrm{~h}$ and sacrificed to assess the effects on peritoneal macrophages, splenocytes and NK cells.

\section{Chemicals}

Unless stated otherwise, all chemicals were purchased from the Sigma Chemical Co. (St Louis, MO). The RPMI 1640 medium and fetal bovine serum (FBS) were purchased from GIBCO (Grand Island, NY). The XTT [2,3-Bis (2methoxy-4-nitro-5-sulfophenyl)-2H-tetrazolium-5-carboxan ilide inner salt] cell viability assay kit was purchased from WelGENE (Daegu, South Korea). All the tissue culture reagents and ethyl acetate fraction from E. bicyclis were assayed for any endotoxin contamination using the Limulus lysate test (E-Toxate, Sigma), and the endotoxin levels were found to be $<10 \mathrm{pg} / \mathrm{ml}$.

\section{Nitrite determination}

Peritoneal macrophages were incubated in either medium alone or medium supplemented for $24 \mathrm{~h}$ and the accumulation of nitrite in culture supernatants was measured using the assay system described by Ding et al., (1988). One hundred micro litter aliquots of culture supernatants were mixed with an equal volume of Griess reagent (mixture at 1:1 of naphthylethylenediamine dihydrochloride and 1\% sulphanilamide in $5 \% \mathrm{H}_{3} \mathrm{PO}_{4}$ ) and incubated at room temperature for $10 \mathrm{~min}$. Nitrite concentration was calculated from a $\mathrm{NaNO}_{2}$ standard curve.

\section{Westem blot analysis for iNOS gene expression}

The amount of iNOS was measured by Western blot analysis. After $24 \mathrm{~h}$ of incubation in 6-well plates, the macrophages were lysed in RIPA buffer $(50 \mathrm{mM}$ Tris- $\mathrm{HCl}$, pH 8.0, $150 \mathrm{mM} \mathrm{NaCl}, 1 \%$ Nonidet-P40, 0.5\% deoxycholic acid, $0.1 \%$ sodium dodecyl sulfate). The protein concent- 
rations were measured using the DC Protein Assay (Bio-Rad Laboratories, Hercules, CA). Twenty micrograms of each sample were electrophoresed on $10 \%$ SDS-PAGE gels and transferred to Hybond-ECL nitrocellulose membranes (Amersham Biosciences, Piscataway, NJ). The membranes were blocked with 5\% skim milk in Tris-buffered saline/ Tween-20 for $1 \mathrm{~h}$. The membranes were incubated with primary antibody against iNOS for $24 \mathrm{~h}$. They were then washed with Tris-buffered saline/Tween-20 once for $15 \mathrm{~min}$ and three times for $5 \mathrm{~min}$, and were incubated with secondary HRP-conjugated anti-rabbit antibody for $1 \mathrm{~h}$. The membranes were then washed again as described above. Autoradiography was carried out using an enhanced chemiluminescence kit (Amersham Bioscience).

\section{Spleen cell proliferative response to mitogen (XTT assay)}

A modification of the method reported by Mosmann et al., (1983) was used. The spleens were aseptically removed and dissociated into a single-cell suspension in a culture medium. Cells ( $5 \times 10^{5}$ cells) were incubated in the presence or absence of the mitogens as follows: concanavalin A (ConA) at $4 \mu \mathrm{g} / \mathrm{ml}$ for $T$ cell activation and lipopolysacchride (LPS) at $10 \mu \mathrm{g} / \mathrm{ml}$ for B cell activation. After incubation of cells for $24 \mathrm{~h}, 20 \mu \mathrm{l}$ of phenazine methosulphate (PMS; electron-coupling reagent) and $25 \mu \mathrm{l}$ of XTT was added to each well. The cells were further incubated for $3 \mathrm{~h}$ to allow XTT formazan production. The absorbance was determined with a microplate reader at a test wavelength of $450 \mathrm{~nm}$ and a reference wavelength of $690 \mathrm{~nm}$.

\section{NK cell tumoricidal activity}

The NK cell tumoricidal activity was carried out in 96-well plates at effecter/target cells ratio 50:1 (rat splenocytes: Yac-1) with $1 \times 10^{4}$ of the target cells in a final well volume of $150 \mu \mathrm{l}$ for $4 \mathrm{~h}$ at $37^{\circ} \mathrm{C}$ in a $5 \% \mathrm{CO}_{2}$ humidified incubator. After incubation, $20 \mu \mathrm{l}$ of PMS and $25 \mu \mathrm{l}$ of XTT was added to each well. The cells were further incubated for $3 \mathrm{~h}$ to allow XTT formazan production. The absorbencies were determined with a microplate reader at a test wavelength of $450 \mathrm{~nm}$ and a reference wavelength of $690 \mathrm{~nm}$.

\section{Statistical analysis}

Each experiment was repeated at least two times, and the results of one representative experiment are shown. The results were expressed as means \pm S.E.M. and analyzed via ANOVA. The significant values are represented by an asterisk $\left({ }^{*}{ }^{*} p<0.05\right)$.

\section{Results and Discussion}

In our pervious study, we showed that the supplementation of EBE reduced body weight in exercise groups. Furthermore EBE has been known to have anti-oxidant effects and antiinflammatory effects (Okada et al., 2004; Whitaker and Carlson, 1975), thus we studied to evaluate immunomodulating effects using innate immune cells to develop EBE as sports nutrition.

Since macrophages and NK cells activation in innate immune system play an important role in the host defense mechanism and NO is related to cytotoxicity and inflammatory functions of macrophages and splenocytes (Bredt and Snyder, 1994; Hibbs et al., 1988), we examined the supplementation effects of $\mathrm{EBE}$ on the production of intermediate regulatory molecule, NO-iNOS by macrophages, NK cells tumoricidal activity and splenocytes proliferative response to mitogen in acute exercise-stress rat model. We showed increasing effects on NO-iNOS production by macrophages and no effects of NK cells tumoricidal activity and the suppressive effects on proliferative response of splenocytes to mitogen in exercise group. However, EBE supplementation in exercise group recovered all these effects such as NO-iNOS production, NK cells tumoricidal activity and the proliferative response of splenocytes to LPS and ConA. Overall, these results suggest that EBE supplementation has differential effects on innate immune response and could be useful as sports nutrition.

As shown in Fig. 1A and Fig. 2, EBE enhanced NO-iNOS production by macrophages, whereas NO production by splenocytes was not altered (Fig. 1B). NO, one of the smallest biological molecular mediators plays important roles in many different aspects of mammalian biology, such as vascular relaxation, neurotransmission, platelet aggregation and immune regulation (Hemmi and Ishida, 1980; Hibbs et al., 1988). Since NO is related to cytostatic or cytotoxic function of macrophages against a variety of tumors (Hibbs et al., 1987; Keller et al., 1990), we examined the effects of EBE 
(a)

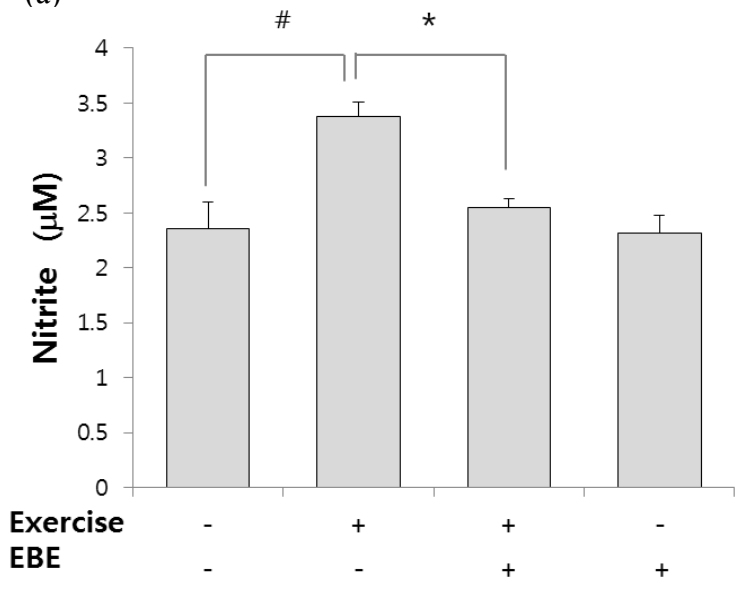

(b)

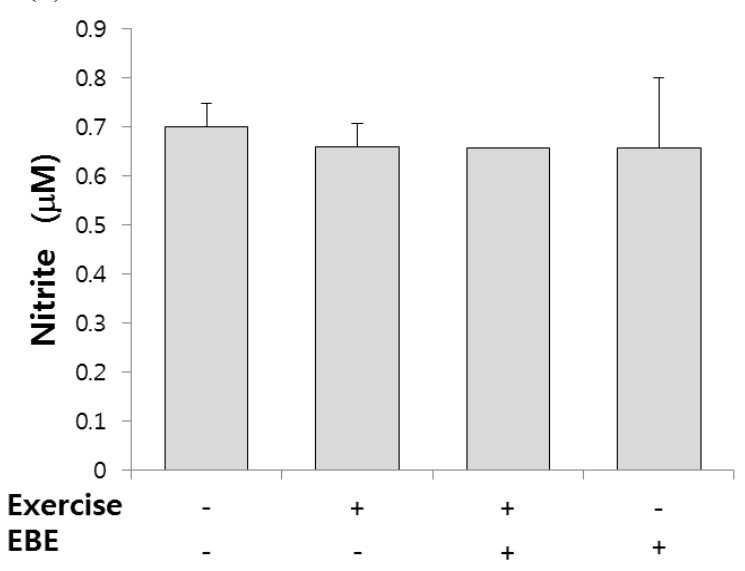

Fig. 1. The effects of the EBE supplementation on the production of NO by (A) peritoneal macrophages and (B) splenocytes from rats. These results are means \pm S.E.M of quintuplicates from a representative experiment. ${ }^{\#} P<0.05$ : significantly different from the untreated control group. ${ }^{*} P<0.05$ : significantly different from the exercise group.

on the NO production from macrophages in exercise-stress rats. It is known that iNOS is expressed in many different cell types and produces high levels of NO. It was produced in high amounts by iNOS in activated macrophages, and then excessive formation of NO plays a key role in inflammatory response of macrophages. Therefore, over-activated macrophages have been known to produce various pro-inflammatory cytokines such as TNF- $\alpha$, IL-1 $\beta$, IL-6 and NO-iNOS that are plays an important roles in inflammatory response. Thus, we also determine the inhibitory effect of EBE on NO-iNOS production in exercise-stimulated macrophages. As shown in Figure 1 and 2, the supplementation of EBE decreased

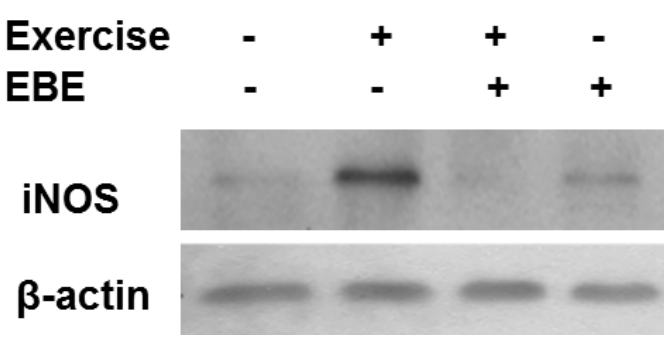

Fig. 2. The inhibitory effects of EBE supplementation on the expression of iNOS in peritoneal macrophages from rats by Western blotting described in methods.

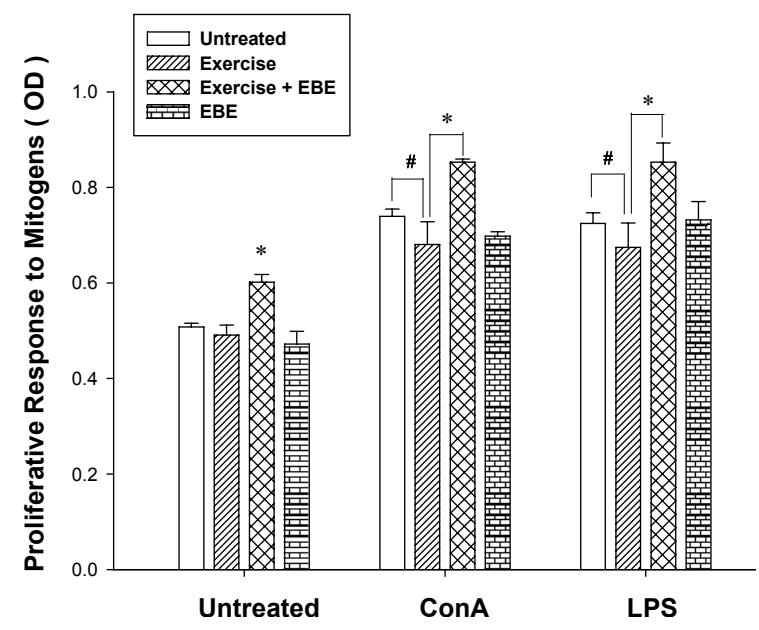

Fig. 3. Effect of the EBE supplementation on the splenocytes proliferative response to mitogen. The splenocytes were incubated in the presence of LPS $(10 \mu \mathrm{g} / \mathrm{ml})$ or ConA $(4 \mu \mathrm{g} / \mathrm{ml})$ for $48 \mathrm{~h}$, and the absorbance was measured at $570 \mathrm{~nm}$. The data is expressed as a means \pm S.E.M of quadruplicates of a representative experiment. ${ }^{\#} P<0.05$ : significantly different from the untreated control group. ${ }^{*} P<0.05$ : significantly different from the exercise group.

NO-iNOS production in exercise-stimulated macrophages.

In the next experiment, to examine the effect of EBE on the splenocytes proliferative response to mitogen, XTT assays were performed by stimulating splenocytes from experimental rats with the mitogens, ConA or LPS for $48 \mathrm{~h}$. To function in adaptive immunity, rare antigen-specific lymphocytes must proliferate extensively before they differentiate into functional effecter cells of a particular immunogenic specificity. Most studies on lymphocyte proliferation have used polyclonal mitogens, which induce many or all lymphocytes of a given type to proliferate in humans (Burleson et al., 1995). These studies indicate that the lymphocyte responses to the T-cell 


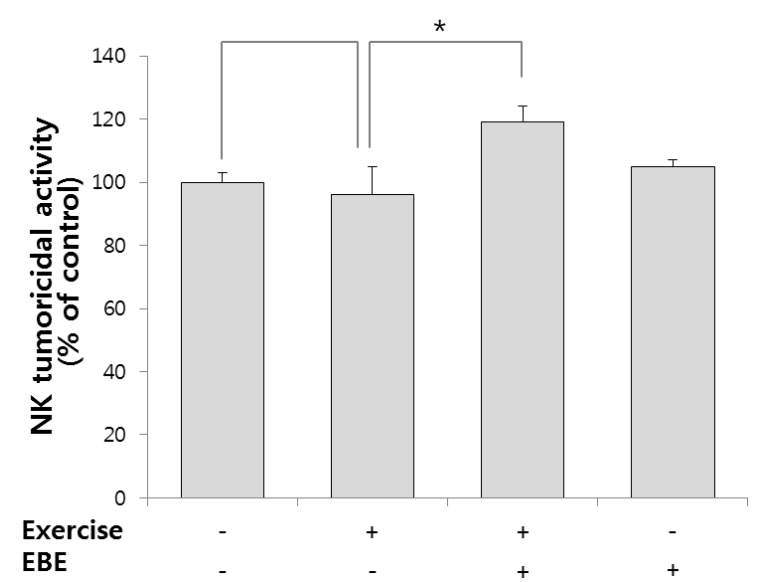

Fig. 4. Effect of the EBE supplementation on the NK cells tumoricidal activity from experimental rats. Splenocytes were cultured with Yac-1 tumor cells for $4 \mathrm{~h}$. The NK cells tumoricidal activity examined at effecter/target cells ratio of $50: 1$ by XTT assay. The data is expressed as a means \pm S.E.M of quadruplicates of a representative experiment. ${ }^{*} P<0.05$ : significantly different from the exercise group.

mitogens ConA decline during and for up to several hours after exercise (Nielsen and Pedersen, 1997; Pedersen and Hoffman-Goetz, 2000), these results have also been a consistent finding in this animal study (Fig. 3). However, administration of EBE increased splenocytes proliferative response to mitogen without any changed effects in EBE intake alone group.

Culturing the splenocytes with tumor target cells (Yac-1) for $4 \mathrm{~h}$, the cytotoxicity of tumor cells were analyzed and resulted in the enhancement of NK cell activity by $119 \%$ in EBE supplementation group compared to the exercise group (Fig. 4). Some reports suggested that the NK cells activity on a per NK cells basis is unchanged during exercise (Nieman $e t$ al., 1993; Palmo et al., 1995) or reduced (Ostrowski et al., 1998b) depending on exercise intensity. These results also a consistent finding in this study and supplementation of EBE in exercise group significantly increased NK cells activity. In NK cells tumoricidal activity and splenocytes proliferative response to mitogen results, there were no effects in EBE intake and maintained at rest groups, however there is increasing effects of NK cells activity and splenocytes proliferation in EBE intake and undergoing exercise groups. These findings provide further evidence for the concept that EBE might be activate the NK cells and splenocytes in priming state which induced by exercise stress.

Collectively, the EBE supplementation inhibits the overproduction of NO-iNOS by macrophages and increased NK cells tumoricidal activity and splenocytes proliferation to mitogens in acute exercise-stimulated rats. These results suggested that EBE supplementation has differential effects on innate immune response and could be useful as sports nutrition.

\section{Acknowledgement}

This work was supported by research fund from Korea Sanhak Foundation (2010). This work was supported by grant No. RTI05-01-02 from the Regional Technology Innovation Program of the Ministry of Knowledge Economy (MKE).

\section{Literature Cited}

Adams, D. O. and T. A. Hamilton. 1984. The cell biology of macrophage activation. Annu. Rev. Immunol. 2:283-318.

Bredt, D. S. and S. H. Snyder. 1994. Nitric oxide: a physiologic messenger molecule. Annu. Rev. Biochem. 63:175-195.

Burleson, G. R., J. H. Dean and A. E. Munson. 1995. Methods in immunotoxicology. Wiley-Liss, New York: 197-210.

Ding, A. H., C. F. Nathan and D. J. Stuehr. 1988. Release of reactive nitrogen intermediates and reactive oxygen intermediates from mouse peritoneal macrophages. Comparison of activating cytokines and evidence for independent production. J. Immunol. 141:2407-2412.

Hemmi, H. and N. Ishida. 1980. The immune response of splenic lymphocytes after cimicifugoside treatment in vitro and pretreatment in vivo. J. Pharmacobiodyn. 3:643-648.

Hibbs, J. B., Jr., R. R. Taintor and Z. Vavrin. 1987. Macrophage cytotoxicity: role for L-arginine deiminase and imino nitrogen oxidation to nitrite. Science 235:473-476.

Hibbs, J. B., Jr., R. R. Taintor, Z. Vavrin and E. M. Rachlin. 1988. Nitric oxide: a cytotoxic activated macrophage effector molecule. Biochem. Biophys. Res. Commun. 157:87-94.

Hoffman-Goetz, L., K. M. May and Y. Arumugam. 1994. Exercise training and mouse mammary tumour metastasis. Anticancer Res. 14:2627-2631.

Hu, T., D. Liu, Y. Chen, J. Wu and S. Wang. 2010. Antioxidant 
activity of sulfated polysaccharide fractions extracted from Undaria pinnitafida in vitro. Int. J. Biol. Macromol. 46: 193-198.

Joe, M. J., S. N. Kim, H. Y. Choi, W. S. Shin, G. M. Park, D. W. Kang and Y. K. Kim. 2006. The inhibitory effects of eckol and dieckol from Ecklonia stolonifera on the expression of matrix metalloproteinase-1 in human dermal fibroblasts. Biol. Pharm. Bull. 29:1735-1739.

Kang, K., H. J. Hwang, D. H. Hong, Y. Park, S. H. Kim, B. H. Lee and H. C. Shin. 2004. Antioxidant and antiinflammatory activities of ventol, a phlorotannin-rich natural agent derived from Ecklonia cava, and its effect on proteoglycan degradation in cartilage explant culture. Res. Commun. Mol. Pathol. Pharmacol. 115-116:77-95.

Keller, R., R. Keist and K. Frei. 1990. Lymphokines and bacteria, that induce tumoricidal activity, trigger a different secretory response in macrophages. Eur. J. Immunol. 20: 695-698.

Madden, K. S. and D. L. Felten. 1995. Experimental basis for neural-immune interactions. Physiol. Rev. 75:77-106.

Mosmann, T. 1983. Rapid colorimetric assay for cellular growth and survival: application to proliferation and cytotoxicity assays. J. Immunol. Methods 65:55-63.

Nielsen, H. B. and B. K. Pedersen. 1997. Lymphocyte proliferation in response to exercise. Eur. J. Appl. Physiol. Occup. Physiol. 75:375-379.

Nieman, D. C., A. R. Miller, D. A. Henson, B. J. Warren, G. Gusewitch, R. L. Johnson, J. M. Davis, D. E. Butterworth and S. L. Nehlsen-Cannarella. 1993. Effects of high- vs moderate-intensity exercise on natural killer cell activity. Med. Sci. Sports Exerc. 25:1126-1134.

Okada, Y., A. Ishimaru, R. Suzuki and T. Okuyama. 2004. A new phloroglucinol derivative from the brown alga Eisenia bicyclis: potential for the effective treatment of diabetic complications. J. Nat. Prod. 67:103-105.

Ostrowski, K., C. Hermann, A. Bangash, P. Schjerling, J. N. Nielsen and B. K. Pedersen. 1998a. A trauma-like elevation of plasma cytokines in humans in response to treadmill running. J. Physiol. 513:889-894.

Ostrowski, K., T. Rohde, S. Asp, P. Schjerling and B. K. Pedersen. 1999. Pro- and anti-inflammatory cytokine balance in strenuous exercise in humans. J. Physiol. 515:287-291.

Ostrowski, K., T. Rohde, M. Zacho, S. Asp and B. K. Pedersen. 1998b. Evidence that interleukin-6 is produced in human skeletal muscle during prolonged running. J. Physiol. 508: 949-953.

Palmo, J., S. Asp, J. R. Daugaard, E. A. Richter, M. Klokker and B. K. Pedersen. 1995. Effect of eccentric exercise on natural killer cell activity. J. Appl. Physiol. 78:1442-1446.

Pedersen, B. K. and L. Hoffman-Goetz. 2000. Exercise and the immune system: regulation, integration, and adaptation. Physiol. Rev. 80:1055-1081.

Sprenger, H., C. Jacobs, M. Nain, A. M. Gressner, H. Prinz, W. Wesemann and D. Gemsa. 1992. Enhanced release of cytokines, interleukin-2 receptors, and neopterin after long-distance running. Clin. Immunol. Immunopathol. 63:188-195.

Whitaker, D. M. and G. P. Carlson. 1975. Anti-inflammation mechanism of extract from Eisenia bicyclis (Kjellman) Setchell. J. Pharm. Sci. 64:1258-1259.

Whiteside, T. L. and R. B. Herberman. 1989. The role of natural killer cells in human disease. Clin. Immunol. Immunopathol. $53: 1-23$

(Received 2 May 2011 ; Accepted 31 May 2011) 